\title{
Research Article \\ Effect of Ultrasonication on Physical Properties of Mineral Trioxide Aggregate
}

\author{
Peter Parashos, Amanda Phoon, and Chankhrit Sathorn \\ Melbourne Dental School, University of Melbourne, 720 Swanston Street, Melbourne, VIC 3010, Australia \\ Correspondence should be addressed to Peter Parashos; parashos@unimelb.edu.au
}

Received 12 February 2014; Accepted 24 February 2014; Published 1 April 2014

Academic Editor: Carla Renata Arciola

Copyright (C) 2014 Peter Parashos et al. This is an open access article distributed under the Creative Commons Attribution License, which permits unrestricted use, distribution, and reproduction in any medium, provided the original work is properly cited.

\begin{abstract}
Aim. To evaluate the effect on physical properties of Mineral Trioxide Aggregate (MTA) of using direct hand compaction during placement and when using hand compaction with indirect ultrasonic activation with different application times. Methods. One hundred acrylic canals were obturated in 3 increments with MTA in sample sizes of 10. One group was obturated by hand with an endodontic plugger and the remainder obturated with indirect ultrasonic application, with times ranging from 2 seconds to 18 seconds per increment. Microhardness values, dye penetration depths, and radiographs of the samples were evaluated. Results. As ultrasonic application time per increment increased, microhardness values fell significantly $(P<0.001)$ while dye penetration values increased $(P<0.001)$. Microhardness of MTA ultrasonicated for 2 seconds was significantly higher than hand compaction $(P=0.03)$. Most radiographic voids were visible in the hand-compacted group $(P<0.001)$, which also had higher dye penetration depths than the 2-second ultrasonicated samples. Ultrasonication of MTA for 10-18 seconds resulted in significantly more voids than 2-8 seconds of ultrasonication $(P=0.02)$. Conclusion. The use of ultrasonics with MTA improved the compaction and flow of MTA, but excessive ultrasonication adversely affected MTA properties. A time of 2 seconds of ultrasonication per increment presented the best compromise between microhardness values, dye penetration depths, and lack of radiographic voids.
\end{abstract}

\section{Introduction}

MTA has grown in popularity as a dental material because of its largely favourable properties, including tissue biocompatibility, superior sealing ability, and its ability to promote dental pulp and periradicular tissue healing [1]. However, there have been concerns amongst clinicians about its difficult handling characteristics. It has been reported that MTA can have different physical and mechanical properties and lose consistency in the presence of excess liquid, which can occur even at the proportion recommended by manufacturers [2] and the resultant mix can lack adequate viscosity [3]. The long setting time of MTA results in an "initial looseness which can make handling rather difficult" [4]. An effective intracanal placement technique of a material such as MTA is imperative, and improving its delivery technique is the key to enhancing MTA's favourable properties [5]. Because there have been few studies examining the variations of placement techniques of MTA, there is little applied consistency amongst its users.
There seems to be limited, and contradictory, information on methods to best handle MTA.

Ultrasonics in dentistry has a wide range of applications and has arguably improved treatment outcomes and predictability [6]. Ultrasonic vibration applied to an endodontic condenser aims to improve the flow, settling, and compaction of MTA and is perceived to be a useful adjunct [7]. However, ultrasonic activation of MTA has been found to result in more voids and poorer adaptation [8]. Furthermore, ultrasonically overcompacted MTA may show poorer physical characteristics because excessive ultrasonication may incorporate air into the MTA and produce a fill less dense and less uniform than that produced by hand compaction [9]. Similarly, ElMa'aita et al. [10] found denser MTA root fillings with manual compaction than with ultrasonic activation. However, regardless of mixing techniques, ultrasonically compacted MTA showed increased compressive strength compared with hand-mixed samples [11]. Ultrasonically compacted MTA produced much higher surface microhardness values than 
manually placed samples [12]. Another study has found significant effect on the push-out bond strength of MTA regardless of mixing methods or ultrasonic application [13].

These different and contradictory findings may be the result of different times used to ultrasonically compact MTA. Therefore, the aim of this study was to evaluate the effect of different ultrasonic application times on the density, compressive strength, and radiopacity of MTA.

\section{Materials and Methods}

Twenty uniform acrylic blocks of five canals each were prepared, creating 100 samples. Each simulated canal measured $1 \mathrm{~mm}$ in diameter by $6 \mathrm{~mm}$ in length. The canals in two of these blocks $(n=10)$ were obturated with MTA without ultrasonic activation. ProRoot MTA (Dentsply Maillefer, Ballaigues, Switzerland) was mixed according to manufacturer's instructions with the supplied sterile water in a 3:1 powder/liquid ratio [14] and hand compacted into the acrylic canals using a similarly sized stainless steel endodontic plugger (American Eagle, Missoula, MT, USA). Each canal was filled in three increments against a glass slab. Both blocks were then placed into a petri dish under cotton pellets soaked with room temperature distilled water and placed in an incubator at $37^{\circ} \mathrm{C}$ for 24 hours at $95 \%$ humidity.

All of the other samples $(n=90)$ were obturated with indirect ultrasonic activation against the endodontic plugger. Samples 11-20 were compacted with 2 seconds of ultrasonic activation, samples 21-30 for 4 seconds, samples 31-40 for 6 seconds, and so forth until samples 91-100, which were ultrasonically activated for 18 seconds. These samples were also filled in three increments, with an ultrasonic tip held lightly against the plugger at each increment for the desired time period. The Cavi 3D tip of the VDW Ultrasonic unit (Aceton, North America, NJ, USA) was used at the middle setting. The MTA fillings were flush with the surface of the acrylic block, and all blocks were placed into petri dishes under cotton pellets soaked with room temperature distilled water. All blocks were then left in an incubator at $37^{\circ} \mathrm{C}$ for 24 hours at $95 \%$ humidity.

\section{Radiographs}

Radiographs were taken for each acrylic block at a set distance of 50 centimeters to the cone of the $\mathrm{X}$ ray unit. The Sirona Heliodent DS X ray unit (Sirona Dental Systems, Bensheim, Germany) was set at $70 \mathrm{kV}$, a current of $7 \mathrm{~mA}$, and an exposure time of 0.25 seconds. These radiographs were then examined for voids, which presented as clearly demarcated radiolucent areas within the specimens. The number of voids, when present, was counted in each specimen.

\section{Microhardness}

The Vickers microhardness was evaluated for the 100 samples. After 24 hours, all the blocks were lightly lapped with 400grit fine sandpaper to produce a smooth surface, and one cement surface per canal was loaded with one Newton for six seconds, with a slope of 0.99 . This resulted in a stamp indent on the MTA surface, with an impression of two orthogonal diagonals. An image of this was brought into focus using the computer and captured immediately after discharge of the diamond indenter. The microhardness of each sample was evaluated using standard calculations [15].

\section{Dye Penetration}

The samples were left in a sealed container for an additional 24 hours after the microhardness testing and immersed in a $0.2 \%$ Rhodamine Blue solution (Ryond Chemical Company, Tianjin, China) for 72 hours in the incubator and then visually assessed for dye penetration. Each of the 100 specimens was examined under a light microscope with $4 \mathrm{x}$ magnification and a Michigan "O" cc periodontal probe ( $\mathrm{Hu}-$ Friedy, Rotterdam, Netherlands) was used to measure the depth of dye penetration. The periodontal probe was held against each specimen and measured to the nearest $0.5 \mathrm{~mm}$.

Statistical analysis included Pearson Correlations, twosample $t$-test, Regression analysis, and Fisher's exact test with significance set at $P<0.05$.

\section{Results}

Evaluation of the microhardness (Figure 1) showed a correlation between increased ultrasonication times and the microhardness of the MTA samples. An approximately linear correlation was demonstrated, with increasing times of ultrasonication resulting in a lower microhardness value ( $r=$ -0.707 , where $r$ is the correlation coefficient; $P<0.001)$. A two-sample $t$-test indicated a significant difference $(P=0.03)$ between hand compaction and two-second ultrasonication. As ultrasonication time increased, the dye penetration of the MTA samples (Figure 2) also increased in an approximately linear manner $(r=0.446, P<0.001)$. As dye penetration increased (Figure 3 ), a corresponding fall in microhardness values was also seen $(P=0.001)$.

When viewing the radiographs taken of the acrylic blocks, a significantly higher proportion $(P<0.001)$ of visible voids were noted in the hand-compacted group (90\%) than in the ultrasonicated groups overall (22\%). Grouping and dichotomising of the ultrasonication groups only indicated a significantly lower proportion $(P=0.02)$ of groups with voids when ultrasonicated for $2-8$ seconds (10\%) than $10-18$ (54\%) seconds.

\section{Discussion}

Hydration of the MTA powder results in a colloidal gel that solidifies to a hard structure in less than three hours, which then continues to set [16]. The basic framework of the hydrated mass is formed by the interlocking of cubic and needle-like crystals in which the needle-like crystals form in sharply delineated thick bundles that fill the intergrain space between the cubic crystals [17]. Its resultant characteristics depend upon liquid additives and quantity [17], as well as entrapped air [18], which may be recognized 


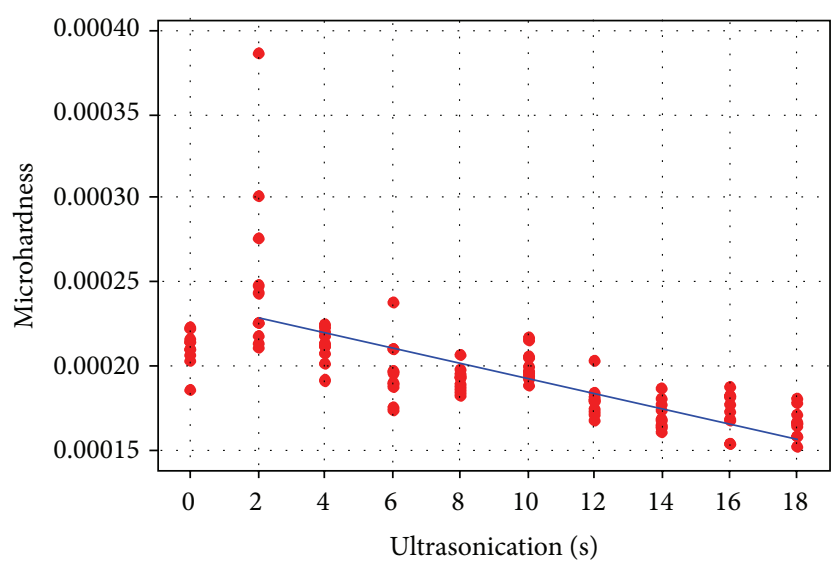

FIGURE 1: Microhardness values and ultrasonication time.

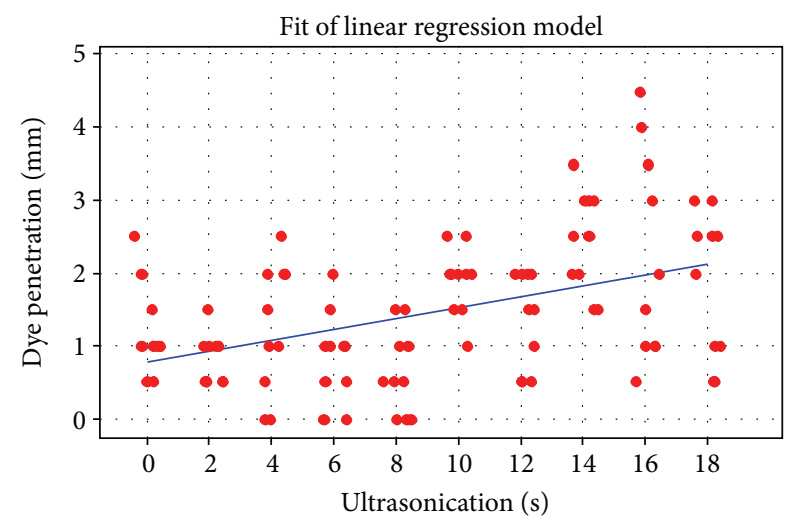

FIGURE 2: Dye penetration and ultrasonication time.

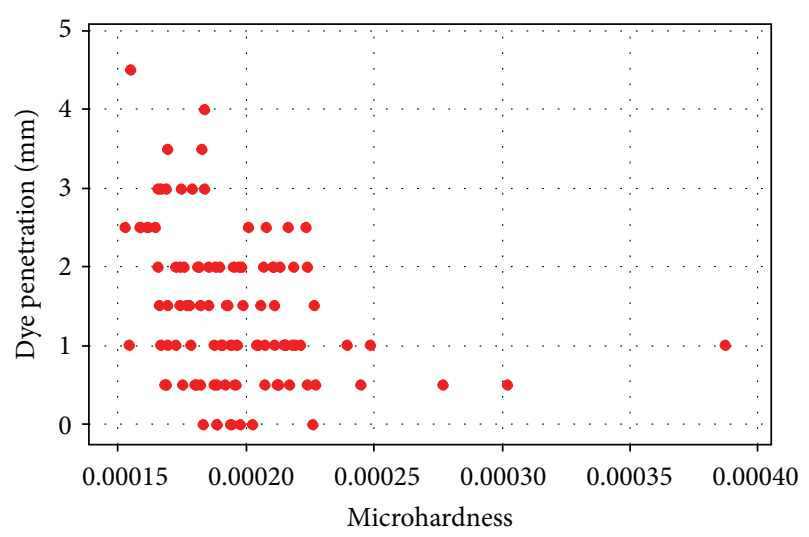

Figure 3: Dye penetration and microhardness.

radiographically. In the present study it was observed that the hand-compacted samples showed more radiographic voids, which was contrary to the findings of Aminoshariae et al. [8]. However, their method differed with the current study because they used $10 \mathrm{~mm}$ lengths of MTA and up to 30 seconds of ultrasonication, although the latter time was not actually specified. Nevertheless, those authors speculated that the reason for their results may have been the length and method of MTA placement [8]. Possibly, the shorter length of MTA and shorter ultrasonication time of the current study were responsible for the better outcome.

In another comparison of hand and ultrasonic compaction [9] ultrasonication produced a denser MTA fill. This study used an ultrasonication time of only one second after their pilot study indicated that up to five-second ultrasonication resulted in radiographically detectable voids. Another recent study [10] used micro-CT analysis and found fewer voids with hand compaction but decreasing number of voids with longer ultrasonication times of five or ten seconds. The present study was unique in assessing a broad range of ultrasonication times, which showed deterioration of the physical properties tested. This may support the phenomenon described by El-Maaita et al. [10] where prolonged activation times may rearrange the MTA particles leading to changes in the numbers of voids, density, and microhardness. Conceivably, the frequency and method of ultrasonication would also exert an effect on the MTA particle arrangement.

The Vickers microhardness test used in this study aimed to quantify the resistance of MTA to plastic deformation. While the compressive strength helps to indicate the setting and hydration reaction progress $[19,20]$, microhardness in itself is not a fundamental material property. In this case, it served to provide a means of assessing the MTA properties between the different samples, and the correlation found was that with increased times of ultrasonication, sample microhardness values fell. Similarly, longer activation time has been found to produce more voids and a lower weight of MTA leading to less dense compactions [9]. This may correlate with the lower microhardness values found in the present study.

The method used for the dye penetration study was adapted from that of Bortoluzzi et al. [21] and aimed to evaluate the resistance of MTA to ingress of fluid. It is recognised that dye studies measuring leakage along root fillings are unreliable and lead to questionable results in extracted teeth [22]. However, in the current study, the amount of dye penetration was used to measure differences of fluid ingress against a smooth plastic wall after different periods of ultrasonication with the aim of assessing potential disturbance of the physical properties of the MTA rather than leakage per se. The hand-compacted samples showed deeper dye penetration. In a similar manner to the microhardness values, with increased ultrasonication of the samples, dye penetration depths rose. This correlates with the number of voids in the MTA, which would influence dye penetration rates. However, it should be noted that the standard deviations in each group were quite wide which may have reflected the measurement method using the periodontal probe and hence some imprecision.

Overall, the results indicated that an ultrasonication time of 2 seconds was better than hand compaction. The higher depth of dye penetration and the higher incidence of radiographic voids amongst the hand-compacted samples indicate that the use of indirect ultrasonic activation is beneficial. It is difficult to directly compare the results of the present study with the available literature because they differ in too many aspects, including type of MTA, the assessment method, 
length of MTA fill, type of canal used, canal dimensions, ultrasonication time, and ultrasonic unit used. Parameters measured also differ and include bacterial leakage [5], pushout strengths [13], solubility $[3,23]$, fracture resistance, and compressive strength $[7,11]$.

\section{Conclusion}

The use of ultrasonics with MTA was useful in improving flow and compaction of MTA, but excessive ultrasonication can adversely impact MTA properties. A suggested time of 2 seconds of ultrasonication per increment presented the best compromise between microhardness values, dye penetration depths, and lack of radiographic voids.

\section{Conflict of Interests}

The authors declare that there is no conflict of interests regarding the publication of this paper.

\section{Acknowledgments}

The authors thank Mr. Ilya Zalizniak for technical assistance and the support of the Melbourne Dental School, the Australian Dental Research Foundation, Gunz Dental (Australia), and Dentsply (Australia).

\section{References}

[1] I. Islam, H. K. Chng, and A. U. J. Yap, "X-ray diffraction analysis of mineral trioxide aggregate and Portland cement," International Endodontic Journal, vol. 39, no. 3, pp. 220-225, 2006.

[2] M. Behr, M. Rosentritt, H. Loher et al., "Changes of cement properties caused by mixing errors: the therapeutic range of different cement types," Dental Materials, vol. 24, no. 9, pp. 11871193, 2008.

[3] M. Fridland and R. Rosado, "Mineral trioxide aggregate (MTA) solubility and porosity with different water-to-powder ratios," Journal of Endodontics, vol. 29, no. 12, pp. 814-817, 2003.

[4] E. S. Lee, "A new mineral trioxide aggregate root-end filling technique," Journal of Endodontics, vol. 26, no. 12, pp. 764-765, 2000.

[5] D. R. Hachmeister, W. G. Schindler, W. A. Walker Jr., and D. D. Thomas, "The sealing ability and retention characteristics of mineral trioxide aggregate in a model of apexification," Journal of Endodontics, vol. 28, no. 5, pp. 386-390, 2002.

[6] G. Plotino, C. H. Pameijer, N. Maria Grande, and F. Somma, "Ultrasonics in endodontics: a review of the literature," Journal of Endodontics, vol. 33, no. 2, pp. 81-95, 2007.

[7] G. R. Lawley, W. G. Schindler, W. A. Walker III, and D. Kolodrubetz, "Evaluation of ultrasonically placed MTA and fracture resistance with intracanal composite resin in a model of apexification," Journal of Endodontics, vol. 30, no. 3, pp. 167-172, 2004.

[8] A. Aminoshariae, G. R. Hartwell, and P. C. Moon, "Placement of mineral trioxide aggregate using two different techniques," Journal of Endodontics, vol. 29, no. 10, pp. 679-682, 2003.

[9] P. Yeung, F. R. Liewehr, and P. C. Moon, "A quantitative comparison of the fill density of MTA produced by two placement techniques," Journal of Endodontics, vol. 32, no. 5, pp. 456-459, 2006.

[10] A. M. El-Ma'aita, A. J. E. Qualtrough, and D. C. Watts, "A microcomputed tomography evaluation of mineral trioxide aggregate root canal fillings," Journal of Endodontics, vol. 38, no. 5, pp. 670-672, 2012.

[11] F. Basturk, M. Nekoofar, M. Gunday, and P. Dummer, "The effect of various mixing and placement techniques on the compressive strength of mineral trioxide aggregate," Journal of Endodontics, vol. 39, pp. 111-114, 2013.

[12] M. H. Nekoofar, Z. Aseeley, and P. M. H. Dummer, "The effect of various mixing techniques on the surface microhardness of mineral trioxide aggregate," International Endodontic Journal, vol. 43, no. 4, pp. 312-320, 2010.

[13] S. Shahi, S. Rahimi, H. R. Yavari et al., "Effects of various mixing techniques on push-out bond strengths of white mineral trioxide aggregate," Journal of Endodontics, vol. 38, no. 4, pp. 501-504, 2012.

[14] H. W. Roberts, J. M. Toth, D. W. Berzins, and D. G. Charlton, "Mineral trioxide aggregate material use in endodontic treatment: A review of the literature," Dental Materials, vol. 24, no. 2, pp. 149-164, 2008.

[15] R. Smith and G. Sandland, "An accurate method of determining the hardness of metals with particular reference to those of a high degree of hardness," Proceedings of the Institution of Mechanical Engineers, vol. 1, pp. 623-641, 1922.

[16] M. Torabinejad, T. F. Watson, and T. R. Pitt Ford, "Sealing ability of a mineral trioxide aggregate when used as a root end filling material," Journal of Endodontics, vol. 19, no. 12, pp. 591-595, 1993.

[17] Y.-L. Lee, B.-S. Lee, F.-H. Lin, A. Yun Lin, W.-H. Lan, and C.P. Lin, "Effects of physiological environments on the hydration behavior of mineral trioxide aggregate," Biomaterials, vol. 25, no. 5, pp. 787-793, 2004.

[18] M. Torabinejad, C. U. Hong, F. McDonald, and T. R. Pitt Ford, "Physical and chemical properties of a new root-end filling material," Journal of Endodontics, vol. 21, no. 7, pp. 349-353, 1995.

[19] M. Torabinejad and N. Chivian, "Clinical applications of mineral trioxide aggregate," Journal of Endodontics, vol. 25, no. 3, pp. 197-205, 1999.

[20] G. Danesh, T. Dammaschke, H. U. V. Gerth, T. Zandbiglari, and E. Schäfer, "A comparative study of selected properties of ProRoot mineral trioxide aggregate and two Portland cements," International Endodontic Journal, vol. 39, no. 3, pp. 213-219, 2006.

[21] E. A. Bortoluzzi, N. J. Broon, C. M. Bramante, R. B. Garcia, I. G. de Moraes, and N. Bernardineli, "Sealing ability of MTA and radiopaque Portland cement with or without calcium chloride for root end filling," Journal of Endodontics, vol. 32, no. 9, pp. 897-900, 2006.

[22] M. K. Wu and P. R. Wesselink, "Endodontic leakage studies reconsidered. Part I: methodology, application and relevance," International endodontic journal, vol. 26, no. 1, pp. 37-43, 1993.

[23] M. Fridland and R. Rosado, "MTA solubility: a long term study," Journal of Endodontics, vol. 31, no. 5, pp. 376-379, 2005. 

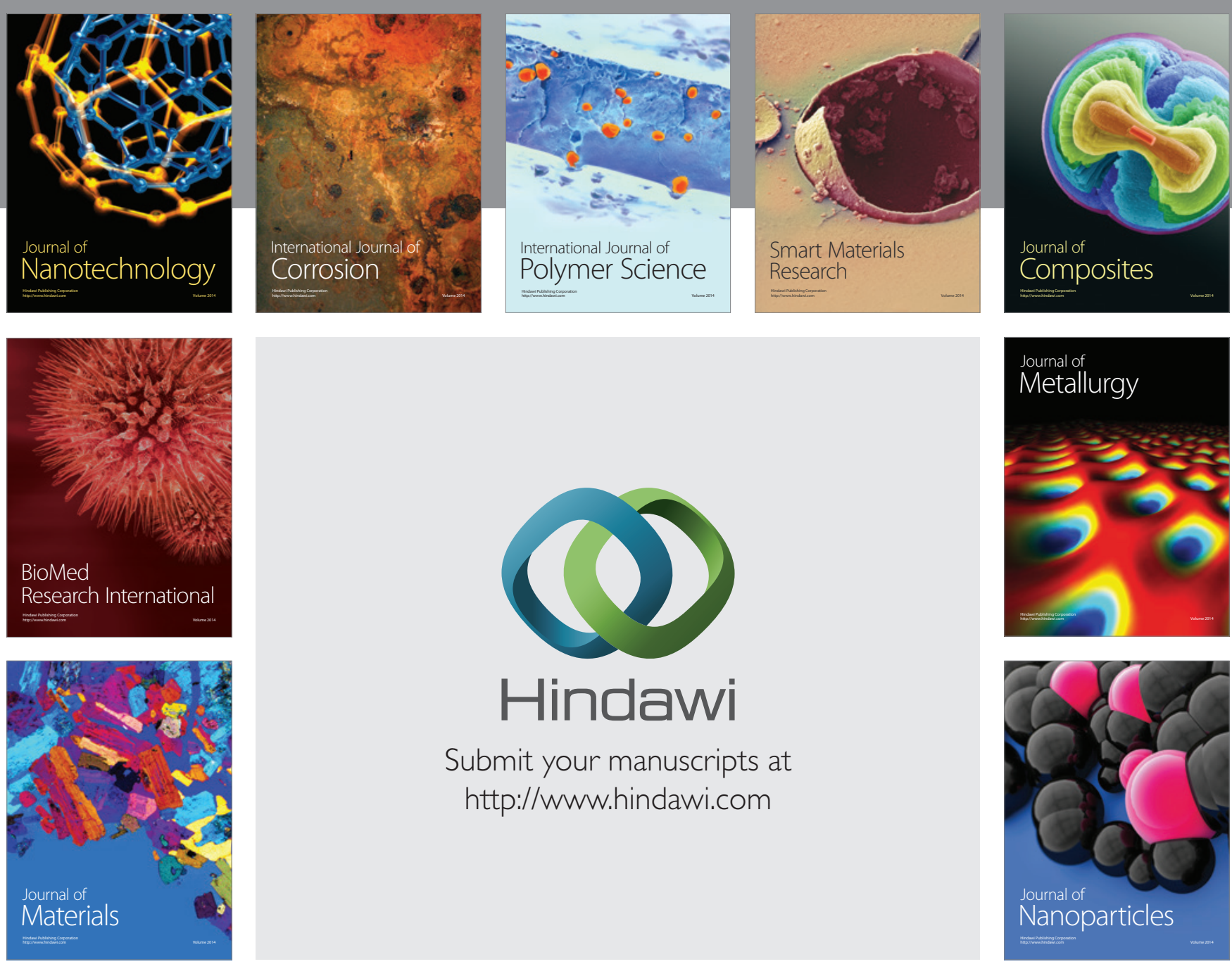

Submit your manuscripts at http://www.hindawi.com
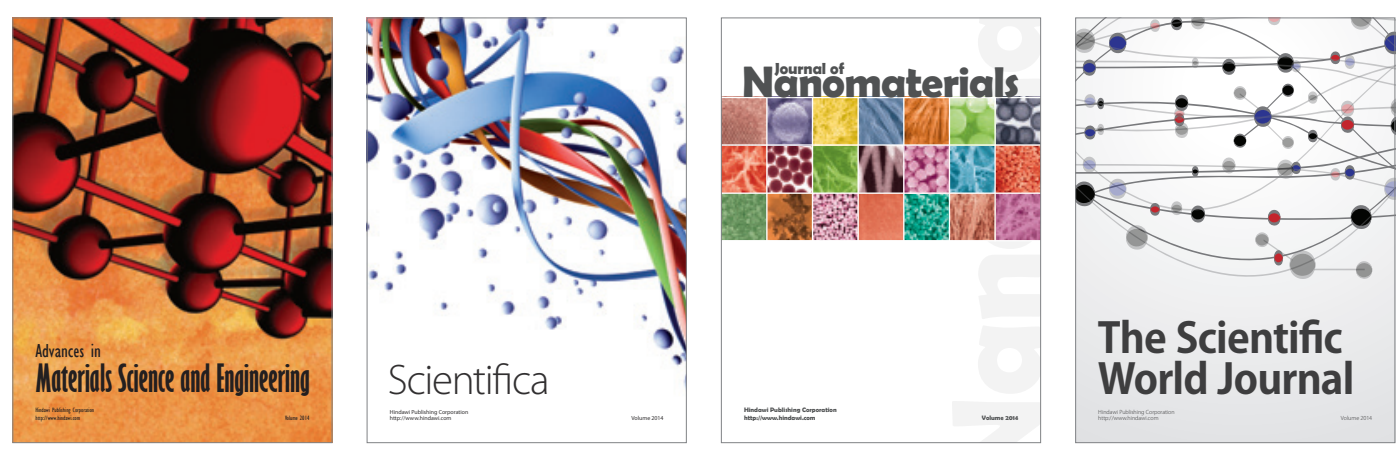

\section{The Scientific World Journal}
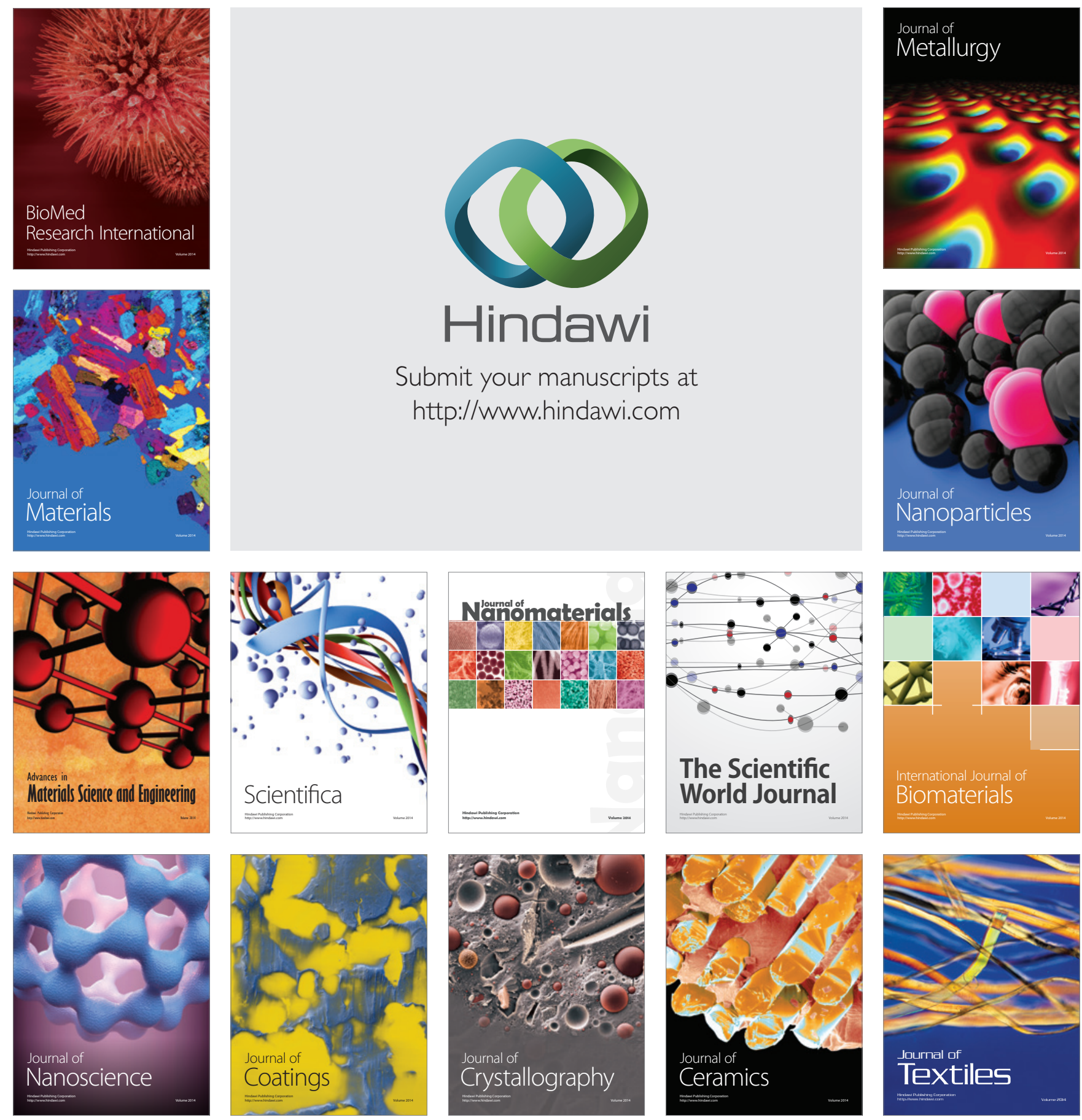